\title{
Spitzer Warm Mission: Maximizing the Science Return in the Extended Mission Phase
}

\author{
Lisa J. Storrie-Lombardi*a, and Suzanne R. Dodd ${ }^{\mathrm{b}}$ \\ ${ }^{a}$ Spitzer Science Center, Infrared Processing and Analysis Center, California Institute of \\ Technology, MS 314-6, Pasadena, USA \\ ${ }^{\mathrm{b}}$ Jet Propulsion Laboratory, 4800 Oak Grove Drive, Pasadena, USA
}

\begin{abstract}
The Spitzer Space Telescope is executing the third observing cycle in the 'warm' extended phase of the mission. For the warm mission, the observatory was effectively reinvented as a new, scientifically productive mission operating at a substantially lower cost. In this paper we describe the ongoing implementation of improvements in science capabilities during the extended mission phase even as the project budget continues to shrink. Improvements in pointing stability, data compression and data analysis techniques allow for new science opportunities more than 8 years after launch. Engineering analyses have shown that the mission can operate with high reliability and minimal technical risk through at least January 2017.
\end{abstract}

Keywords: Spitzer Space Telescope, Operations, Extended Mission

\section{INTRODUCTION}

The Spitzer Space Telescope, NASA's Great Observatory for infrared astronomy, was launched 25 August 2003[1]. Spitzer provides sensitivity that is almost three orders of magnitude greater than that of any previous ground-based and space-based infrared observatory. The five and half year cryogenic mission was successfully completed in May of 2009. Upon depletion of the cryogen, the instrument chamber warmed to $\sim 27 \mathrm{~K}$. At this new equilibrium temperature, the 3.6 and 4.5 micron bands of the InfraRed Array Camera (IRAC, PI: Giovanni Fazio, SAO[2]) maintain their cryogenic mission performance levels. Science operations for the warm mission began July 28, 2009 and the observatory is now executing Cycle 8, the third cycle of warm observations. The annual operations budget, including data analysis support for the astronomical community, has been reduced by two-thirds from a level of $\sim \$ 72$ million in FY08 to $\$ 22-24$ million today.

We describe here the efforts made to continue maximizing the observing efficiency and scientific productivity during the extended mission phase even as the project budget continues to shrink. Recent improvements in pointing stability, data compression and data analysis techniques allow for new science opportunities more than 8 years after launch. The observatory continues to execute cutting edge science and remains NASA's most efficient community observatory with more than $90 \%$ of the time spent executing science observations. There are no identified engineering obstacles to continuing operations into at least 2017. The planning and implementation of the transition from cryogenic to warm mission operations has been discussed in previous papers (e.g. [3], [4]). In this paper we highlight the efforts made to maximize the science return during the warm mission.

\section{EFFICIENT OPERATIONS}

To maximize the science output from a limited lifetime mission, observing efficiency is a driving requirement. This influenced the final design of the observatory, instruments and operations concepts. Even though the cryogen has been depleted, several of these design choices help maximize warm operations efficiency.

*email: lisa@,ipac.caltech.edu, srdodd@,jpl.nasa.gov

URLs: http://ssc.spitzer.caltech.edu// http://www.spitzer.caltech.edu/

Observatory Operations: Strategies, Processes, and Systems IV, edited by

Alison B. Peck, Robert L. Seaman, Fernando Comeron, Proc. of SPIE Vol. 8448

84481E - @ 2012 SPIE - CCC code: 0277-786X/12/\$18 - doi: 10.1117/12.925249

Proc. of SPIE Vol. 8448 84481E-1 


\subsection{Instruments}

To maximize the science output from a cryogenic mission with a limited lifetime, observing efficiency is a driving requirement. This influenced the final design of the observatory, instruments and operations concepts. Even though the cryogen has been depleted, several of these design choices help maximize efficiency in the extended mission. There was no science benefit to be gained during the cryogenic mission from operating the instruments in parallel as this would just deplete the cryogen more quickly. However, the instruments were designed so that 'internal parallel' observations were available in most observing modes. The IRAC instrument takes data in the 3.6 and 4.5 micron channels simultaneously, even though they do not share the same field of view. The Spitzer instruments are shown in Figure 1. IRAC is the black instrument in the lower right.

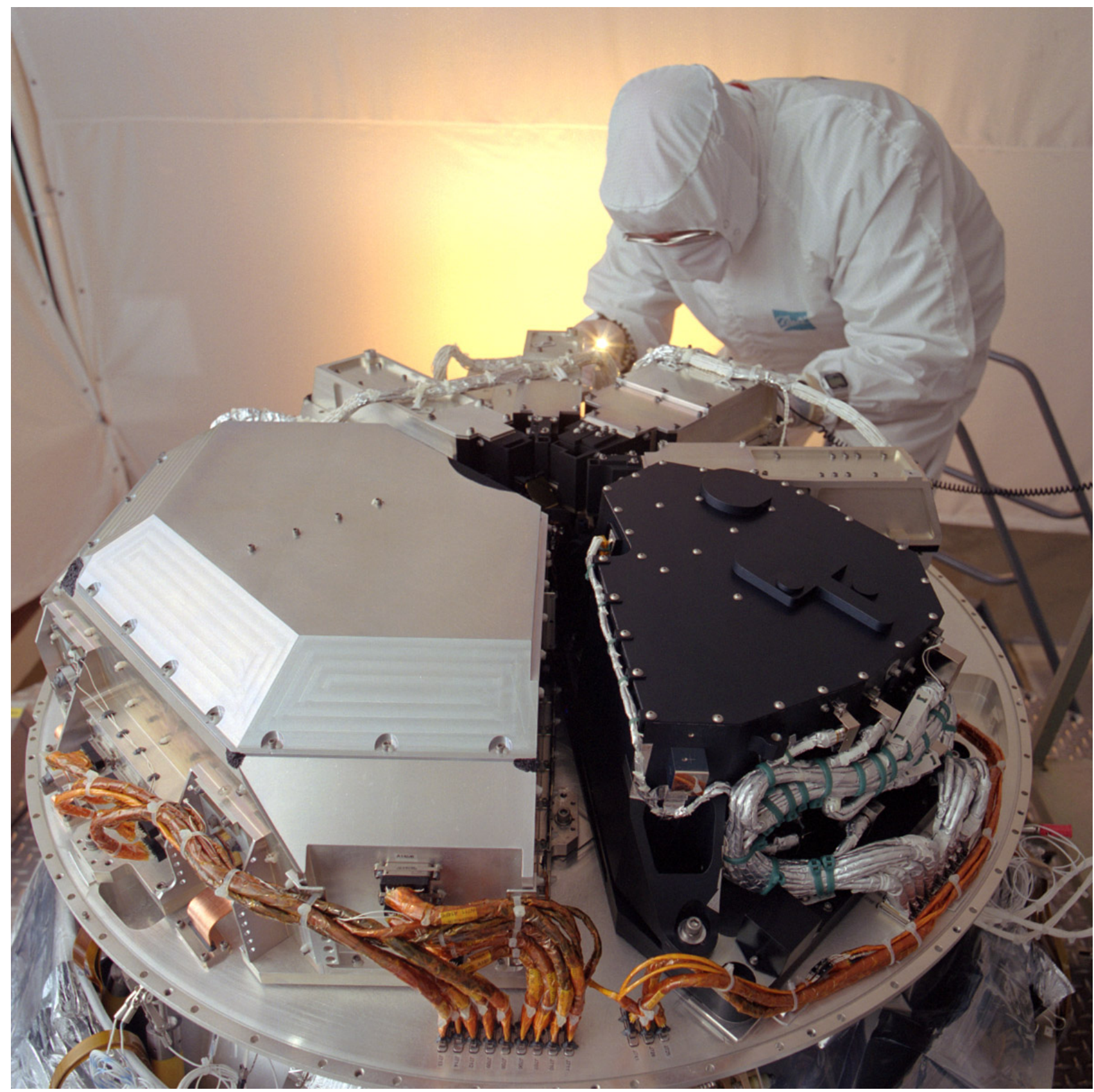

Figure 1. The three Spitzer instruments are the IRAC (black, lower right), MIPS (silver, lower left) and the IRS (rear). 


\subsection{Observing Modes}

The fundamental unit of Spitzer observing is the Astronomical Observing Request (AOR). It is composed of the selected instrument, channel, dithering and mapping parameters chosen from an Astronomical Observation Template (AOT), combined with the targeting information. AOTs provide a limited but powerful set of options for observers to use in crafting their science program. The fact that the combination of options is a finite set gives the observatory a well defined set of modes to calibrate. There are no 'orphan modes', e.g. a grating rarely used or a unique exposure time that requires special calibration. Calibration is robust and special extra calibrations are rarely required.

\subsection{Command Generation}

Providing estimates of the observing time required is of course essential in the proposal planning phase. The software engine that provides the resource estimates for Spitzer is the same software that eventually builds the command products. This means that the estimates are of extremely high fidelity and makes a one-phase proposal process possible for most programs. The original mission plan was completely a one-phase process but this was relaxed, based on experience, so that very large, complex programs did not need to provide a complete set of AORs with the proposal. Science user support is still required to help investigators craft their programs to meet their science goals but after the proposal selection is complete, most of the programs are fully defined. Spitzer Science Center (SSC) resources can then be devoted to the complex and/or large programs that require more attention.

\subsection{Observing Cadence and Scheduling}

The mission is scheduled in one-week blocks with a new master sequence controlling the observations for an entire week. The weeks are divided into periods of autonomous operation (PAOs), which are defined as the time between spacecraft contacts. Science observations do not continue during the spacecraft contacts because the observatory orientation is selected to point the high-gain antenna at the earth. During the cryogenic mission the spacecraft contacts were every 12 to 24 hours and during the warm mission they are every 24 to 48 hours. Non-science observatory activities are minimal and the uplink of sequences and commands and the downlink of the data occur between the PAOs. This results in 20 to 22 hours/day for science observations. The scheduling process takes just over 5 weeks from the start of implementation on the ground to the start of execution on board. Additional time was added to the early part of the scheduling process on the ground for warm operations. See [5] and [6] for more details on the scheduling process. A schematic of the process is shown in Figure 2. No late changes are made to the approved schedules except for the health and safety of the observatory. Observer errors are not corrected now if discovered after the observing schedule as been approved which occurs $\sim 3$ weeks before execution begins. This has been made repeatedly clear to the observers and requests for late changes have dramatically decreased during the warm mission.

\subsection{Programs Supported}

It was recognized early in the planning for the warm mission that greater resource reductions would have to be made in the science operations than the mission operations. Even though the observatory was losing two instruments it would still be taking data 24 hours a day, 365 days a year. The only obvious tasks that changed for mission operations was that we no longer needed to monitor or manage the cryogen and the number of spacecraft contacts would be halved. For science operations, support for the MIPS[7] and IRS[8] instruments was no longer required. The MIPS and IRS teams at the SSC comprised less than $20 \%$ of the total staffing. Therefore other major changes were required to obtain a substantial reduction in operating costs. The SSC supported $\sim 250$ observing programs a year and this has been reduced to $\sim 60$ programs annually during the warm mission. This was accomplished with substantial community input [9] that resulted in a new program category of called Exploration Science (ES). ES proposals are $>500$ hours is size and have opened up science opportunities that were not available during the cryogenic mission. The Exploration Science programs selected in the most recent cycle are listed in Table 1.

\subsection{Proposal Process}

Many observatories use a two-phase proposal process, where a science proposal is submitted and peer-reviewed, and only the successful proposers then submit a detailed second phase proposal specifying the details of the observations. Spitzer has used a hybrid process throughout the mission. Small proposals are required to completely specify their observations in their original proposal. The AOTs (discussed in section 2.2) make this feasible for small to moderate 
sized-programs. Large programs are allowed to submit their science proposal with representative AORS, but they don't have to submit the complete set of AORs until after the proposal is successful. The limited user support resources at the Spitzer Science Center can then be devoted to the programs with the most challenging science programs and/or complex AORs.

During the cryogenic mission, Spitzer held annual in-person proposal reviews involving 100 external reviewers spending 3-5 days in Pasadena to accomplish the review of $\sim 800$ proposals. For the warm mission we now receive $150-200$ proposals per cycle and the panel reviews are carried out by telecon, followed up by a face-to-face TAC meeting of 8 people. This requires substantially fewer resources to support.

Table 1. The Cycle-8 Exploration Science programs.

\begin{tabular}{|c|c|c|c|c|c|}
\hline ID & $\begin{array}{l}\text { Science } \\
\text { Category }\end{array}$ & $\begin{array}{l}\text { PI } \\
\text { Institution }\end{array}$ & Title & Co-Is & Hours \\
\hline 80025 & $\begin{array}{l}\text { nearby } \\
\text { galaxies }\end{array}$ & $\begin{array}{l}\text { Liese van Zee } \\
\text { University of Indiana }\end{array}$ & $\begin{array}{l}\text { Stellar Distributions in Dark Matter Halos: } \\
\text { Looking Over the Edge }\end{array}$ & 7 & 1005 \\
\hline 80057 & $\begin{array}{l}\text { high-z } \\
\text { galaxies }\end{array}$ & $\begin{array}{l}\text { Giovanni Fazio } \\
\text { Smithsonian Astrophysical Obs. }\end{array}$ & $\begin{array}{l}\text { Spitzer Very Deep Survey of the } \\
\text { HST/CANDELS Fields }\end{array}$ & 12 & 1182 \\
\hline 80096 & $\begin{array}{l}\text { high-z } \\
\text { clusters }\end{array}$ & $\begin{array}{l}\text { S. Adam Stanford } \\
\text { U. C. Davis }\end{array}$ & SPT-Spitzer Deep Field & 33 & 766 \\
\hline 80100 & $\begin{array}{l}\text { high-z } \\
\text { galaxies }\end{array}$ & $\begin{array}{l}\text { Casey Papovich } \\
\text { Texas } A \& M\end{array}$ & $\begin{array}{l}\text { Spitzer-HETDEX Exploratory Large Area } \\
\text { (SHELA) Survey }\end{array}$ & 28 & 526 \\
\hline 80072 & $\begin{array}{l}\text { nearby } \\
\text { galaxies }\end{array}$ & $\begin{array}{l}\text { Brent Tully } \\
\text { University of Hawaii }\end{array}$ & Cosmic Flows & 7 & 200 \\
\hline 80040 & YSOs & $\begin{array}{l}\text { John Stauffer } \\
\text { Spitzer Science Center }\end{array}$ & $\begin{array}{l}\text { YSOVARII: Mapping YSO Inner Disk Structure } \\
\text { in NGC2264 - Simultaneous Spitzer +CoRoT }\end{array}$ & 40 & 630 \\
\hline 80073 & exoplanets & $\begin{array}{l}\text { Heather Knutson } \\
\text { Caltech }\end{array}$ & $\begin{array}{l}\text { Life on the Edge: Planetary Atmospheres in } \\
\text { Extreme Environments }\end{array}$ & 12 & 596 \\
\hline 80117 & exoplanets & $\begin{array}{l}\text { David Charbonneau } \\
\text { Harvard University }\end{array}$ & $\begin{array}{l}\text { Validating the First Habitable-Zone Planet } \\
\text { Candidates Identified by Kepler }\end{array}$ & 13 & 600 \\
\hline 80179 & $\begin{array}{l}\text { brown } \\
\text { dwarfs }\end{array}$ & $\begin{array}{l}\text { Stanimir Metchev } \\
\text { SUNY Stony Brook }\end{array}$ & $\begin{array}{l}\text { Weather on Other Worlds: A Survey of Cloud- } \\
\text { Induced Variability in Brown Dwarfs }\end{array}$ & 9 & 873 \\
\hline 80016 & exoplanets & $\begin{array}{l}\text { Jessica Krick } \\
\text { Spitzer Science Center }\end{array}$ & Comparative Atmospheric Study of Exoplanets & 13 & 619 \\
\hline 80074 & $\begin{array}{l}\text { galactic } \\
\text { structure }\end{array}$ & $\begin{array}{l}\text { Barbara Whitney } \\
\text { University of Wisconsin }\end{array}$ & $\begin{array}{l}\text { Deep GLIMPSE: Exploring the Far Side } \\
\text { of the Galaxy }\end{array}$ & 51 & 600 \\
\hline
\end{tabular}




\section{Nominal Uplink Process Timeline Overview}

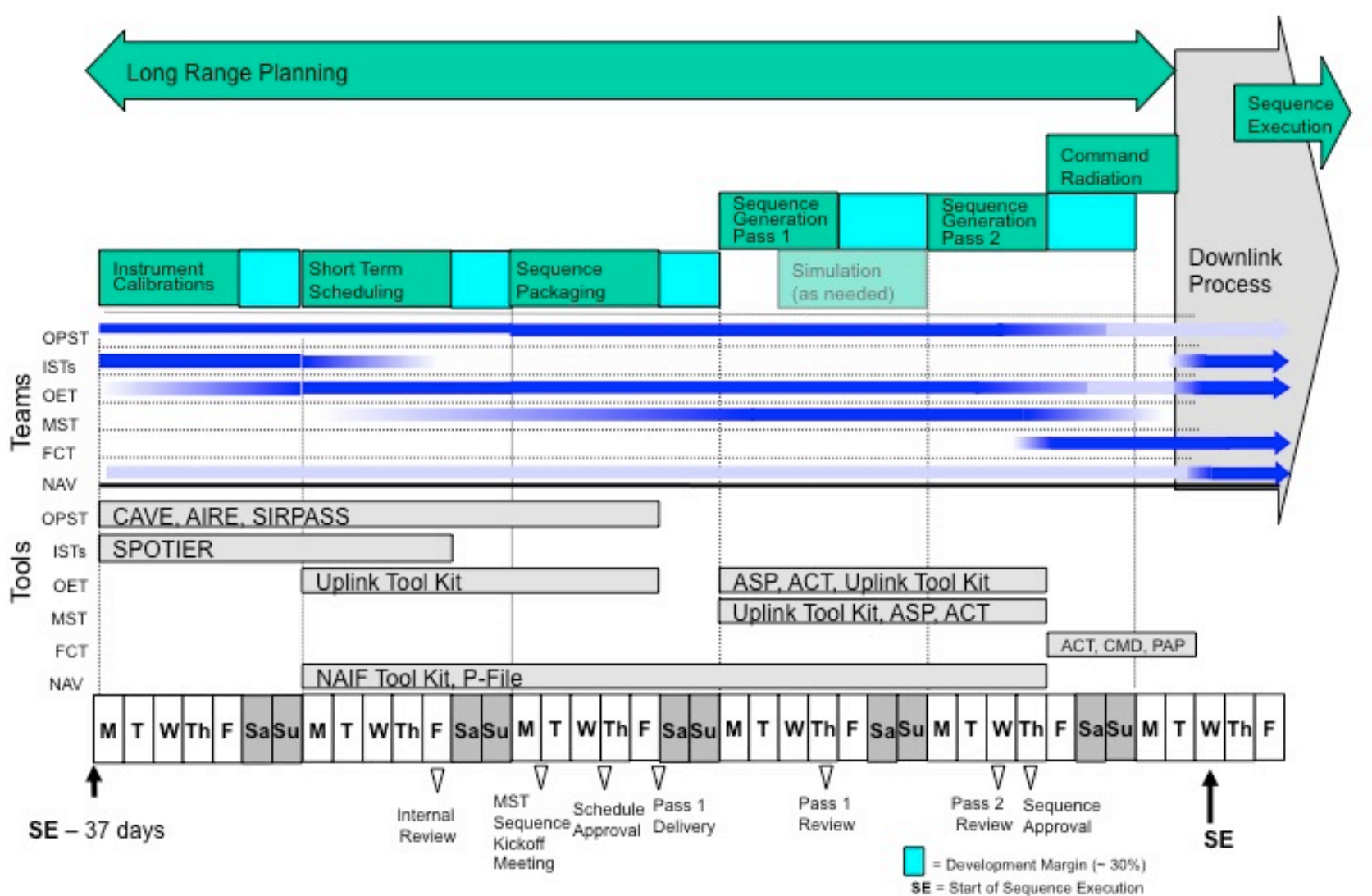

Figure 2. The nominal five-six week scheduling process in shown here. The observatory scheduling is described in detail in the references [5] and [6].

\section{SCIENCE DRIVEN MISSION ENHANCEMENTS}

The science and mission operations teams continue to work closely to further improve the observatory operations and the science data analysis, in particular to support one of Spitzer's key science strengths - the characterization of exoplanets. Over $25 \%$ of the time allocated in warm mission has been devoted to science requiring high-precision relative photometry, including exoplanet characterization and investigations of cloud cover and weather for brown dwarfs. Spitzer observations typically reach noise levels within $20 \%$ of the photon-noise limit in the unbinned time series. Precisions of $100 \mathrm{ppm}$ are routinely reached, and more recently, precisions of $30 \mathrm{ppm}$ have been achieved [10]. Spitzer's excellent photometric precision enables a wide range of exoplanet science including validation of candidates, measuring planetary radii, characterizing atmospheric composition and thermal lapse rates, and determining the day-night atmospheric temperature difference.

Extracting high-precision photometry from the data is complicated by sources of correlated noise, which must be trended from the data[12]. The most significant source of correlated noise is variations in the gain across individual detector pixels coupled to small telescope motions during the long-staring integrations used for exoplanet observations. Due to the sharpness of the Spitzer point spread function relative to the pixel size, small telescope motions during an observation cause the peak of the observed brightness function to be sampled by different subpixel positions. Since the gain can vary by up to $8 \%$ across a given pixel, small motions introduce a small though significant photometric variation. The photometric variations are a source of correlated noise in the photometric time series. Both the telescope and instrument perform far better than the design specification and the amplitude of the correlated noise is typically a 
fraction of a percent. These observations are typically $8-24$ hours in length and the longest staring observations to date have been 3-4 weeks in duration. Therefore pointing stability and data volume management are key issues.

\subsection{High Precision Photometry}

The IRAC observing modes have been optimized for the warm mission. Additional exposure times have been added to the high-dynamic range mode as well as to the sub-array observing modes. The most significant enhancements have been made to support high precision photometry and are summarized in Table 2 . They are:

1. Reduce spacecraft pointing variations resulting from a sawtooth-shaped periodic wobble, which had an amplitude of 0.15 arcseconds and a period of one hour prior to October 2010.

2. Place the targets more accurately on the desired position on the 'sweet-spot' pixel. This is accomplished using a new mode call PCRS Peak-up that improves pointing accuracy and repeatability.

3. Improved calibration products to allow more sophisticated data analysis techniques using high resolution gain maps.

Table 2. High Precision Photometry Enhancements.

\begin{tabular}{|c|c|c|c|}
\hline Action & Result & Observations Enabled & Implemented \\
\hline $\begin{array}{l}\text { Heater cycling } \\
\text { increased to reduce } \\
\text { pointing wobble. }\end{array}$ & $\begin{array}{l}50 \% \text { reduction in amplitude } \\
\text { of photometric wobble due } \\
\text { to intrapixel gain variation } \\
50 \% \text { reduction in wobble } \\
\text { period }\end{array}$ & $\begin{array}{l}\text { Transit measurements improved } \\
\text { through } 50 \% \text { smaller correlated } \\
\text { noise signatures and moving wobble } \\
\text { period away from transit durations } \\
\text { Exoplanet science observations } \\
\text { improved through reduced } \\
\text { systematics }\end{array}$ & October 2010 \\
\hline $\begin{array}{l}\text { Use of PCRS Peak- } \\
\text { up to place stars } \\
\text { precisely on IRAC } \\
\text { arrays. }\end{array}$ & $\begin{array}{l}\text { Pointing repeatability } \\
\text { improves from } 0.3 \text { arcsec } \\
\text { rms to } 0.08 \text { arcsec rms } \\
\text { Position source on region } \\
\text { of minimal gain variation } \\
\text { resulting in a two to five } \\
\text { times reduction in } \\
\text { correlated noise }\end{array}$ & $\begin{array}{l}\text { Permits longitudinal temperature } \\
\text { profiles of exoplanets using sparsely } \\
\text { sampled observations, doubling } \\
\text { number of planets surveyed } \\
\text { - Improves full-phase light curves } \\
\text { through mitigation of pointing drift } \\
\text { - Improves precision of exoplanet data } \\
\text { and facilitates co-addition of } \\
\text { multiple transits by placing targets } \\
\text { on position of minimal gain variation } \\
\text { - Improves precision of exoplanet data } \\
\text { via development of intra-pixel gain } \\
\text { map }\end{array}$ & $\begin{array}{l}\text { December } \\
2011\end{array}$ \\
\hline $\begin{array}{l}\text { Independent, facility- } \\
\text { provided intra-pixel } \\
\text { gain map. }\end{array}$ & $\begin{array}{l}\text { Better than } 100 \text { ppm map } \\
\text { of intra-pixel gain } \\
\text { Removes systematic } \\
\text { uncertainly inherent with } \\
\text { self calibration }\end{array}$ & $\begin{array}{l}\text { Transit measurements improved } \\
\text { through } 50 \% \text { smaller correlated } \\
\text { noise signatures and moving wobble } \\
\text { period away from transit durations } \\
\text { Exoplanet science observations } \\
\text { improved through reduced } \\
\text { systematics }\end{array}$ & $\begin{array}{l}\text { March - } \\
\text { September } \\
2012\end{array}$ \\
\hline
\end{tabular}

Pointing Wobble: The pointing wobble was correlated with the cycling of a heater that maintains the battery near a constant temperature. The battery is located near the position where the star-tracker attaches to the spacecraft bus. The pointing wobble is thought to result from thermal warping of this attachment point, causing the tracker boresight to wander in phase with the heater cycling. The heater ON/OFF range was initially set to $2 \mathrm{~K}$ (range of 4 data numbers). A 
thermal analysis revealed no issues with reducing the cycling range to $2 \mathrm{DNs}$ or $1 \mathrm{~K}$ and this was implemented in October 2010. As a result, the heater cycles with half the amplitude and double the frequency. Reducing the heater amplitude had the desired result of reducing the amplitude of the pointing wobble by $50 \%$ as well as reducing the period of oscillation to $\sim 36$ minutes. The reduction in pointing wobble amplitude cuts the photometric variation in half and shifts the spectral power of the wobble further away from the period of planetary transits and eclipses (typically an hour or more). The shift in period makes aliasing of exoplanet signatures less likely. This is shown graphically in Figure 3.

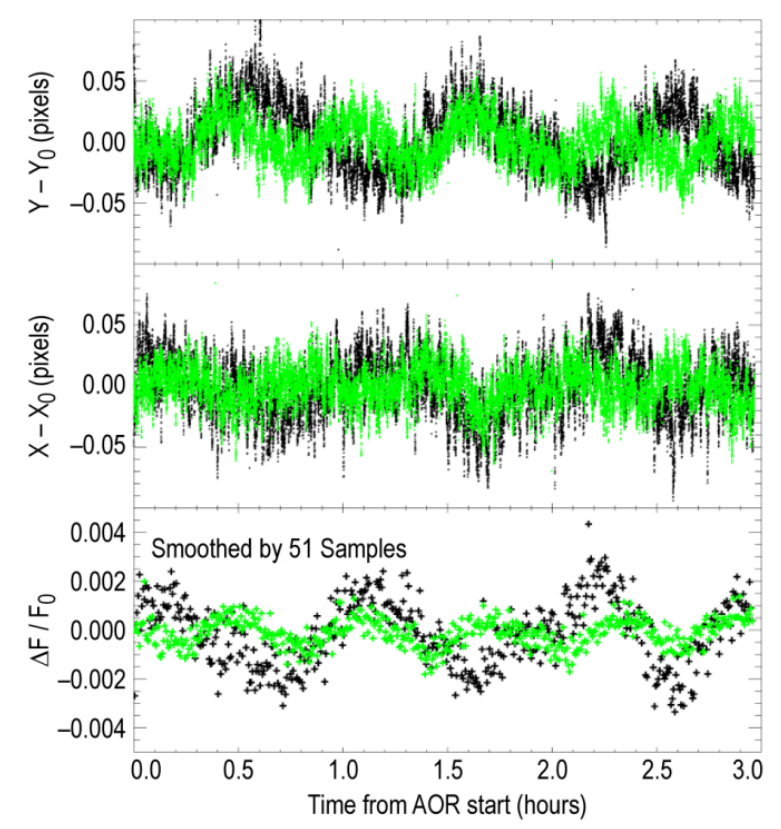

Figure 3. The pointing centroid positions ( $y=$ top panel; $x=$ middle panel) and the photometric wobble (bottom panel) for identical $4.5 \mu \mathrm{m}$ observations of the standard, HD 158460, with the original heater cycling shown in black and the reduced heater cycling shown in green. The reduced heater range produces a $50 \%$ reduction in correlated noise.

PCRS Peak-Up: The PCRS Peak-up mode was implemented in late 2011 and provides enhanced accuracy in positioning a target on a specific part of an identified pixel which and can improve greatly the photometric precision in staring mode observations. The mode uses the Pointing Calibration and Reference Sensor (PCRS) on the observatory. The PCRS was used to peak-up on sources for the IRS instrument during the cryogenic mission. The PCRS operates in the visual part of the spectrum (505-595 nanometers) and its main function is to calibrate and remove the optical offset between the star trackers and the telescope. The PCRS can measure the centroids of stars in the magnitude range $7.0<\mathrm{V}$ $<12.5$ to an accuracy of better than 0.14 " (1 sigma radial). Because of intra-pixel sensitivity variations the measured flux of a point source varies by about $8 \%$ in the $3.6 \mu \mathrm{m}$ array (Channel 1) and $4 \%$ in the $4.5 \mu \mathrm{m}$ array (Channel 2), depending on where the target centroid falls on a pixel. This "pixel phase effect" is the most significant source of correlated noise in staring mode observations. PCRS peak-up allows the positioning (and repositioning for multiple observations) of a target to an accuracy of about 0.1 pixel, reducing the range of the pixel phase effect.

Intra-pixel Gain Maps: In parallel to implementing the PCRS Peak-up as an observing mode, the IRAC Instrument support team at the SSC is building high precision maps of the intra-pixel photometric variation in a region approximately $1 / 4$ pixels on a side, centered on the intra-pixel gain "sweet spots" (positions of maximum sensitivity) for the 3.6 and 4.5 micron sub-array fields of view. These maps will enable the intra-pixel gain correction of staring mode observations of point sources without using the science observations themselves. For details see Ingalls, et al., this volume[11].

\subsection{Data Volume and Compression}

Spitzer is in an earth-trailing orbit and continues to drift further away from the earth every year. The rate at which data can be downlinked is now $550-1100 \mathrm{bps}$, due the increased distance from the earth, compared to $2.2 \mathrm{Mbps}$ during the 
bulk of the cryogenic mission. The amount of data that can be stored on the mass memory card on board is also limited. For its primary full-array observing mode, IRAC nominally takes data in both the 3.6 and 4.5 -micron channels. Data taking for one channel can be turned off for observations where the second channel is not scientifically useful and data volume is an issue.

The IRAC and Observatory Engineering Teams have designed an implemented two different modifications in the data compression strategies (Table 3) that lead to $20-40 \%$ improvements in the data compression. These enable longer staring observations of bright targets using short exposure times, as well as larger area shallow surveys.

Table 3. Data Compression Improvements.

\begin{tabular}{|l|l|l|c|}
\hline \multicolumn{1}{|c|}{ Action } & \multicolumn{1}{c|}{ Result } & \multicolumn{1}{c|}{ Observations Enabled } & Implemented \\
\hline $\begin{array}{l}\text { Offset modification } \\
\text { to Rice compression. }\end{array}$ & $\begin{array}{l}\text { Previously incompressible } \\
\text { data compresses by } 40 \% .\end{array}$ & $\begin{array}{l}\text { Longer stares of brighter exoplanets } \\
\text { and larger area shallow surveys. }\end{array}$ & November 2011 \\
\hline $\begin{array}{l}\text { Drop least significant } \\
\text { bit of IRAC data. }\end{array}$ & $\begin{array}{l}\text { Improves data compression of } \\
\text { all science data by } \sim 20 \% .\end{array}$ & $\begin{array}{l}\text { Higher data volume observations at } \\
\text { lower downlink rates and higher } \\
\text { cadence observations. }\end{array}$ & $\begin{array}{c}\text { Dec } 2011 \text { to } \\
\text { July } 2012\end{array}$ \\
\hline
\end{tabular}

\section{SUMMARY}

The Spitzer Space Telescope continues to deliver forefront science that addresses all of the major themes of Astro2010 Decadal Survey. Now in the third year of our extended warm mission, the project continues to implement improvements to maximize the science return from the observatory. This paper provides an overview and several more detailed Spitzer papers from this conference are summarized below.

Mahoney, W.A., et al., "Spitzer operations: scheduling the out years", PAPER 8448-76 [6]

Ingalls, J. G., et al., "Intra-Pixel Gain Variations and High-Precision Photometry with the Infrared Array Camera (IRAC)", PAPER 8442-68 [11]

Grillmair, C.J., et al., "Pointing Effects and Their Consequences for Spitzer IRAC Exoplanet Observations", PAPER $8448-47$ [12]

Krick, J.E., et al., “A Spitzer IRAC Measure of the Zodiacal Light”, PAPER 8442-67 [13]

Lowrance, P.J., et al., "Modifications to the Warm Spitzer data reduction pipeline", PAPER 8442-117 [14]

Carey, S.J et al., "Absolute photometric calibration of IRAC: lessons learned using nine years of flight data", PAPER $8442-69$ [15]

Hora, J.L., et al., “The IRAC point response function in the Warm Spitzer Mission”, PAPER 8442-118 [16]

\section{REFERENCES}

[1] Werner, M., Roellig, T., Low, F., Rieke, G., Rieke, M., Hoffmann, W., Young, E., Houck, J., Brandl, B., Fazio, G., Hora, J., Gehrz, R., Helou, G., Soifer, B., Stauffer, J., Keene, J., Eisenhardt, P., Gallagher, D., Gautier, T., Irace, W., Lawrence, C., Simmons, L., Van Cleve, J., Jura, M., Wright, E., “The Spitzer Space Telescope Mission”, ApJS, $154,1,(2004)$

[2] Fazio, G. G., Hora, J. L., Allen, L. E., Ashby, M. L. N., Barmby, P., Deutsch, L. K., Huang, J.-S., Kleiner, S., Marengo, M., Megeath, S. T., Melnick, G. J., Pahre, M. A., Patten, B. M., Polizotti, J., Smith, H. A., Taylor, R. S., Wang, Z., Willner, S. P., Hoffmann, W. F., Pipher, J. L., Forrest, W. J., McMurty, C. W., McCreight, C. R., McKelvey, M. E., McMurray, R. E., Koch, D. G., Moseley, S. H., Arendt, R. G., Mentzell, J. E., Marx, C. T., Losch, P., Mayman, P., Eichhorn, W., Krebs, D., Jhabvala, M., Gezari, D. Y., Fixsen, D. J., Flores, J., Shakoorzadeh, K., Jungo, R., Hakun, C., Workman, L., Karpati, G., Kichak, R., Whitley, R., Mann, S., Tollestrup, E. V., Eisenhardt, P., Stern, D., Gorjian, V., Bhattacharya, B., Carey, S., Nelson, B. O., Glaccum, W. J., Lacy, M., Lowrance, P. J., Laine, S., Reach, W. T., Stauffer, J. A., Surace, J. A., Wilson, G., Wright, E. L., Hoffman, A., 
Domingo, G., Cohen, M., “The Infrared Array Camera (IRAC) for the Spitzer Space Telescope”, ApJS, 154, 10 (2004)

[3] Storrie-Lombardi, L.J. and Dodd, S.R., "Downsizing a great observatory: reinventing Spitzer in the warm mission," in Proceedings of the SPIE - Observatory Operations: Strategies, Processes, and Systems III. Edited by Silva, David R.; Peck, Alison B.; Soifer, B., Volume 7737: 77370L-77370L-9 (2010).

[4] Dodd, Suzanne R., Storrie-Lombardi, Lisa, Scott, Charles P., "Spitzer's model for dealing with the end of the cryogenic mission," in Proceedings of the SPIE - Observatory Operations: Strategies, Processes, and Systems II. Edited by Brissenden, Roger J., Silva, David R., Volume 7016, 70160D-70160D-9 (2008).

[5] Mahoney, W.A., Garcia, L.J., Hunt, Jr., J., McElroy, D.B., Mannings, V.G., Mittman, D.S., O’Linger, J.C., Sarrel, M., Scire, E., "Spitzer warm mission transition and operations," Proc. SPIE, Volume 7737: 77371W-77371W-12 (2010).

[6] Mahoney, W.A., Effertz, M.J., Fisher, M.E., Garcia, L.J., Hunt, Jr., J.C., Mannings, V., McElroy, D.G., Scire, E. et al., "Spitzer operations: scheduling the out years", Proc. SPIE, THIS VOLUME, 8448-76 (2012)

[7] Rieke, G., and E. T. Young, C. W. Engelbracht, D. M. Kelly, F. J. Low, E. E. Haller, J. W. Beeman, K. D. Gordon, J. A. Stansberry, K. A. Misselt, J. Cadien, J. E. Morrison, W. B. Latter, A. Noriega-Crespo, D. L. Padgett, K. R. Stapelfeldt, D. C. Hines, E. Egami, J. Muzerolle, A. Alonso-Herrero, M. Blaylock, H. Dole, J. L. Hinz, E. Le Floc'h, C. Papovich, P. G. Perez-Gonzalez, P. S. Smith, K. Y. L. Su, L. Bennett, D. T. Frayer, D. Henderson, N. Lu, F. Masci, M. Pesenson, L. Rebull, J. Rho, J. Keene, S. Stolovy, S. Wachter, W. Wheaton, P. L. Richards, "The Multiband Imaging Photometer for Spitzer", ApJS, 154, 25 (2004)

[8] Houck, J., Roellig, T., van Cleve, J., Forrest, W., Herter, T., Lawrence, C., Matthews, K., Reitsema, H., Soifer, B., Watson, D., Weedman, D., Huisjen, M., Troeltzsch, J., Barry, D., Bernard-Salas, J., Blacken, C., Brandl, B., Charmandaris, V., Devost, D., Gull, G., Hall, P., Henderson, C., Higdon, S., Pirger, B., Schoenwald, J., Sloan, G., Uchida, K., Appleton, P., Armus, L., Burgdorf, M., Fajardo-Acosta, S., Grillmair, C., Ingalls, J., Morris, P., Teplitz, H., "The Infrared Spectrograph on the Spitzer Space Telescope", ApJS, 154, 18 2004)

[9] Storrie-Lombardi, L. J., and Silbermann, N. A., "The Science Opportunities of the Warm Spitzer Mission Workshop," AIP Conference Proceedings 943; Conference Location and Date: Pasadena, California, 4-5 June 2007.

[10] Gillon, M., Demory, B.-O., Benneke, B., Valencia, D., Deming, D., Seager, S., Lovis, Ch., Mayor, M., Pepe, F., Queloz, D., Ségransan, D., Udry, S., "Improved precision on the radius of the nearby super-Earth 55 Cnc e", A\&A, 539, 28 (2012)

[11] Ingalls, J.G., Krick, J.E., Carey, S.J., Laine, S., Surace, J.A., Glaccum, W.J., Grillmair, C.J., Lowrance, P.J., "IntraPixel Gain Variations and High-Precision Photometry with the Infrared Array Camera (IRAC)", Proc. SPIE, THIS VOLUME, 8442-68 (2012)

[12] Grillmair, C.J., Carey, S.J., Stauffer, J.R., Fisher, M.E., Olds, R., Ingalls, J.G., Krick, J.E., Glaccum, W.J., Laine, S., Surace, J.A., "Pointing Effects and Their Consequences for Spitzer IRAC Exoplanet Observations", Proc. SPIE, THIS VOLUME, 8448-47 (2012)

[13] Krick, J.E., Glaccum, W.J., Carey, S.J., Lowrance, P.J., Surace, J.A., Ingalls, J.G., Hora, J.L., Reach, W.T., “A Spitzer IRAC Measure of the Zodiacal Light", Proc. SPIE, THIS VOLUME, 8442-67 (2012)

[14] Lowrance, P.J., Carey, S., Krick, J., Glaccum, B., Khan, I., Ingalls, S., Laine, S., Grillmair, C., "Modifications to the Warm Spitzer data reduction pipeline", Proc. SPIE, THIS VOLUME, 8442-117 (2012)

[15] Carey, S.J., Ingalls, J.G., Hora, J.L., Surace, J.A., Glaccum, W.J., Lowrance, P.J., Krick, J.E., Cole, D.M., Laine, S., Engelke, C.W., Price, S.D., Bohlin, R.C., Gordon, K.D., "Absolute photometric calibration of IRAC: lessons learned using nine years of flight data", Proc SPIE, THIS VOLUME, 8442-69 (2012)

[16] Hora, J.L., Marengo, M., Park, R., Wood, D., Hoffman, W.F., Lowrance, P.L, Carey, S.J., Surace, J.A., Krick, J.E., Glaccum, W.J., Ingalls, Laine, S., Fazio, G.F., Willner, S.P., Ashby, M.L.N., Wang, Z., "The IRAC point response function in the Warm Spitzer Mission", Proc SPIE, THIS VOLUME, 8442-118 (2012) 